\title{
AVALIAÇÃO DA QUALIDADE DO PROCESSO DE DESINFECÇÃO EM SUPERFÍCIES INANIMADAS DE UNIDADES BÁSICAS DE SAÚDE POR PESQUISA DE BIOMARCADORES
}

FUCCI, Ana Paula Bandeira; MARCOLINO, Moniele Storti. Discentes do curso de Farmácia UNIFEV. CASTRO, Valéria da Cruz Oliveira de; REZENDE, Catia. Docentes do curso de Fármacia UNIFEV. Rua Pernambuco, n. ${ }^{\circ} 4196$ - Centro - Votuporanga-SP. E-mail: catia_rezende@terra.com.br.

\begin{abstract}
Resumo
A Infecção Relacionada à Assistência à Saúde - IRAS pode ocorrer pela transmissão exógena por meio da contaminação de superfícies contaminadas. A presente pesquisa teve como objetivo verificar a qualidade do processo de desinfecção de superfícies inanimadas de Unidades Básicas de Saúde - UBS de um município do noroeste paulista, através da presença de Staphylococcus aureus e Escherichia coli. Foram avaliadas sete UBSs, em horários e dias aleatórios, abrangendo as seguintes superfícies: maçaneta da sala de curativos, torneiras e bebedouros, mesa do consultório, guichê da recepção. Swabs estéreis foram friccionados em $20 \mathrm{~cm}^{2}$ das superfícies e transportados em meio Stuart ao Laboratório Didático de Análises Clínicas do UNIFEV. As amostras foram cultivadas em Ágar Sangue e MacConkey, a 35 \pm 1 oC por 24 horas, emjarra de microaerofilia e aerobiose, respectivamente. O Staphylococcus aureus foi identificado por meio da produção de hemolysin, catalalase and coagulase. Já a Escherichia coli, via os seguintes testes bioquímicos: TSI,citrato urease, indole, lysine, ornithine and arginina. Das 105 amostras analisadas, 6,66\% apresentaram positivas para S. aureus e 2,85\% para E. coli. As superfícies que apresentaram a presença dos bioindicadores foram: o guichê da recepção, guichê da farmácia, maçaneta da sala de curativos, torneira da sala de curativos e bebedouro. Esses resultados corroboram outros estudos que demonstram que as superfícies inanimadas são importantes fontes de contaminação no ambiente de assistência à saúde, contribuindo com a contaminação cruzada e, consequentemente, com o aumento de infecção ao paciente que está sujeito aos procedimentos nesse contexto. Dentro desse contexto, o poder público, através das políticas públicas de saúde, é responsável pela capacitação dos profissionais da saúde, contribuindo na promoção e prevenção da saúde coletiva.
\end{abstract}

Palavras-Chave: Staphylococcus aureus; Escherichia coli; Superfícies; IRAS; Biomarcadores.

AN EVALUATION OF THE QUALITY OF THE DESINFECTION PROCESS IN INANIMATED SURFACES OF BASIC HEALTH UNITS BY BIOMARKERS RESEARCH

\begin{abstract}
Infection Related Health Care - IRHC may occur by exogenous transmission through the contamination of contaminated surfaces. This study aimed at verifying the quality of the process of disinfecting inanimate surfaces of Basic Health Units - BHU in a northeastern city in São Paulo state, through the presence of biomarkers, Staphylococcus aureus and Escherichia coli. We evaluated 7 UBS in random times and days, covering the following areas: dressing-room doorknob, drinking fountains and faucets, office desk, reception counter. Sterile swabs were rubbed on a $20 \mathrm{~cm}^{2}$ surface and transported to the laboratory in Stuart medium to the Clinical Analyses Didactic Laboratory of UNIFEV. The samples were cultured on Blood agar and MacConkey agar at $35 \pm 1$ oC for 24 hours in aerobic and microaerophilic jar, respectively. Staphylococcus aureus was identified by the production of hemolysin, catalase and coagulase. Escherichia coli was identified using the biochemical tests: TSI, citrate, urease, indole, lysine, ornithine and arginine. Of the 105 samples analyzed, $6.66 \%$ of the
\end{abstract}


samples were positive for Staphylococcus aureus and Escherichia coli to $2.85 \%$. The Areas which showed the presence of biomarkers were: the reception booth, booth pharmacy, handles of the dressing room, dressing room faucet and drinking fountain. These results corroborate other studies that show that inanimate surfaces are important sources of contamination in the healthcare environment, contributing to crosscontamination and, consequently, to the increase of infection to the patient who is subjected to procedures in this environment. Within this context, government, by means of public health policies, is responsible for the training of health professionals, contributing to the promotion and prevention of public health

KEYWORDS: Staphylococcus aureus; Escherichia coli; Surfaces; IRHA; Biomarker.

\section{INTRODUÇÃO}

Infecção Relacionada à Assistência à Saúde - IRAS são infecções adquiridas após a admissão do paciente ao hospital, que se podem manifestar durante sua internação ou após sua alta, desde que estejam relacionadas com algum procedimento realizado durante sua permanência no hospital. Também podem ser relacionadas com procedimentos que foram realizados em ambulatórios, consultórios e outras unidades ligadas ao atendimento a saúde (BRASIL, 1998).

A IRAS no Brasil é um importante problema de saúde pública, gerando dificuldades sociais e econômicas, tanto em países desenvolvidos como em países em desenvolvimento, ocasionando um aumento significativo na morbidade, na mortalidade e nos custos hospitalares (WENZEL, 1992; RABHAE, 2000; BOYCE, 1997).

O ambiente em estabelecimentos de saúde, que inclui o ar, a água e as superfícies inanimadas que cercam o paciente, guarda íntima relação com as Infecções Relacionadas à Assistência a Saúde, podendo proporcionar focos de contato e de transmissão de agentes patogênicos (ANDRADE et al., 2000).

Quando as normas de Biossegurança (Lei 8974/ 1995) não são seguidas adequadamente, os equipamentos utilizados e materiais se tornam extremamente perigosos, levando os profissionais ligados diretamente à sua rotina, e também os visitantes, vítimas pela exposição aos agentes contaminantes e nocivos à saúde (CARVALHO, 1999).

As mãos contaminadas dos funcionários atuam como um importante meio de disseminação no mecanismo de transmissão de infecção nos estabelecimentos de saúde, onde os artigos de múltiplos usos acabam se tornando veículos de agentes infecciosos, quando não sofrerem os processos de descontaminação após cada uso. Os locais e as pessoas onde estes artigos são preparados podem tornar-se fontes de infecção para os hospedeiros suscetíveis. Uma das importantes fontes de contaminação são o contato direto com fluidos corpóreos, durante a realização de procedimentos que sejam considerados invasivos ou por meio da manipulação de algum artigo como roupas, lixo e até mesmo as superfícies contaminadas, sem que se utilizem as medidas de biossegurança (BRASIL, 1994; SEQUEIRA, 2001).

Os agentes patogênicos podem ser transferidos para as mais diversas superfícies através do contato direto com as mãos, equipamentos, respingos de sangue e saliva, micro-organismos do meio ambiente entre outros, carregados pelos indivíduos presentes neste local. Nas últimas décadas acabou se levantando a questão da importância do ambiente como um grande reservatório de micro-organismos multirresistentes, onde estes podem agravar o quadro de pacientes suscetíveis (TEIXEIRA, VALLE, 1996; SHIOMORI et al., 2002).

Itens hospitalares se tornam um grande reservatório de patógenos, que promovem a contaminação cruzada do estabelecimento de saúde e, assim, a contaminação das mãos, justificando a sua limpeza e desinfecção adequadas (CARVALHO, 2005).

O homem também pode infectar-se por meio da poeira infecciosa, que pode ser originada de fonte humana ou ambiental: através do espirro ou do ato de tossir, quando se expelem gotículas que vão se depositar nas superfícies, de onde evaporam deixando 
resíduos; a movimentação de lençol e o ato de varrer o assoalho, que faz com que esses resíduos fiquem circulantes no ar junto com o micro-organismo que pode sobreviver por longos períodos nessa poeira, em ambientes de pouca ventilação, o que acaba criando um perigo significativo em hospitais, clínicas, consultórios odontológicos, pois contribui para a disseminação de várias doenças. As infecções transmitidas pelo ar podem ser restritas ao trato respiratório ou podem acometer outras áreas do corpo (MELO et al., 2004).

O Staphylococcus aureus permanece como um importante patógeno envolvido à etiologia das IRAS, podendo sobreviver por meses em amostras clínicas secas, sendo resistente ao calor e elevadas concentrações de sal. Os indivíduos sadios são colonizados desde a amamentação e podem albergar esse micro-organismo na nasofaringe, ocasionalmente na pele e muito raramente na vagina. A partir desses sítios, pode-se contaminar a pele e membranas mucosas do paciente, objetos ou até outros pacientes por contato direto ou aerossol, ocasionando infecções por conta do seu fator de virulência ou através de sua resistência aos antimicrobianos utilizados atualmente (BRASIL, 2004; MUNDIM et al., 2003).

A E. coli faz parte da microbiota humana, presente no trato gastrointestinal, e é comumente causadora de sepse, meningites neonatais, infecções do trato urinário e gastroenterites; a maioria dessas infecções são endógenas, exceto a meningite neonatal e gastroenterite (PRËRE, FAYET, 2005).

Dentro desse contexto, o objetivo deste estudo foi realizar a avaliação da qualidade do processo de desinfecção nas superfícies inanimadas de Unidades Básicas de Saúde, por pesquisa de biomarcadores, já que a biossegurança, por meio da adoção de normas e procedimentos seguros, visa à manutenção da saúde dos pacientes; assim, faz-se necessária esta avaliação (SCHEIDT et al., 2006).

\section{Metodologia}

No período de junho a outubro de 2012 foram analisadas sete Unidades Básicas de Saúde - UBS do
Noroeste Paulista. As UBS estavam localizadas em bairros periféricos da cidade, sendo uma UBS considerada de grande porte, com pronto-atendimento, onde se realizavam pequenas cirurgias. As outras realizam procedimentos relacionados às diversas especialidades: atendimento ginecológico, pediátrico, geral, odontológico, grupo para diabéticos, hipertensos, terceira idade, Programa Viva Leite, saúde de ferro entre outros. As crianças e os idosos eram os pacientes mais frequentes nas unidades avaliadas.

Em seis UBSs foram avaliadas as seguintes superfícies inanimadas: guichê da recepção, guichê da farmácia, maçaneta da sala de curativos, torneira da sala de curativos e bebedouro. Na unidade de grande porte foram avaliados maçaneta da sala de medicação, mesa da sala de medicação, torneira da sala de medicação, maçaneta da sala de pequenas cirurgias e bebedouro. Essas coletas foram realizadas através da fricção, em $20 \mathrm{~cm}^{2}$ da superfície investigada, com swabs estéreis. Os mesmos foram acondicionados em meio de transporte Stuart e caixa isotérmica, encaminhados ao Laboratório Didático de Análises Clínicas da UNIFEV. As amostras foram cultivadas em Ágar Sangue e MacConkey incubadas a $35 \pm 1^{\circ} \mathrm{C}$ por 24 horas em jarra de microaerofilia e aerobiose, respectivamente. Após a incubação foi realizada a confirmação macroscópica, por meio da hemolisina, e microscópica das colônias, através do método de Gram. Posteriormente, as colônias suspeitas de $S$. aureus foram submetidas às provas da catalase, coagulase e sal Manitol. Para confirmação de colônias suspeitas de E. coli foram realizadas as provas bioquímicas: TSI, Citrato de Simmons, Urease, Lisina, Ornitina, Arginina e Indol.

\section{Resultados e discussão}

Cada uma das sete UBS's foi avaliada em três meses diferentes, em dias e horários aleatórios. Dessa maneira, foram avaliadas 15 amostras por unidade, totalizando 105 amostras.

Das 105 amostras, 6,7\% (7/105) foram positivas para Staphylococcus aureus e 2,9\% (3/105) para Escherichia coli. Ao correlacionarmos os locais 
analisados e as porcentagens de contaminação pelos biomarcadores, foram verificados índices semelhantes na torneira da sala de curativos, guichê da farmácia, maçaneta da sala de curativos e bebedouro (Tabela 1).

Tabela 1 - Correlação das superfícies avaliadas e frequência de biomarcadores em 105 amostras analisadas.

\begin{tabular}{ccc}
\hline Staphylococcus aureus & $\begin{array}{c}\text { RESULTADO } \\
\text { POSITIVO }\end{array}$ & $\begin{array}{c}\text { VALOR EM } \\
\text { PORCENTAGEM }\end{array}$ \\
\hline Torneira da Sala de Curativos & $2 / 7$ & $28,6 \%$ \\
Guichê da Rece pção & $1 / 7$ & $14,3 \%$ \\
Guichê da Farmácia & $2 / 7$ & $28,6 \%$ \\
Maçaneta da Sala de Curativos & $2 / 7$ & $28,6 \%$ \\
\hline
\end{tabular}

\section{Escherichia coli}

\begin{tabular}{ccc}
\hline Bebedouro & $2 / 7$ & $28,6 \%$ \\
Torneira & $1 / 7$ & $14,3 \%$ \\
\hline
\end{tabular}

Fonte: Dados de pesquisa.

Raros dados da literatura demonstram pesquisa de bioindicadores realizadas em Unidades Básicas, sendo frequentes os estudos que abordam o ambiente hospitalar.

O índice de contaminação demonstrado neste estudo está muito abaixo de dados relatados em pesquisas envolvendo superfícies inanimadas no ambiente hospitalar.

Oie e colaboradores (2002) relataram $27 \%$ de amostras positivas para $S$. aureus em maçanetas de enfermarias. Carvalho (2005) realizou uma pesquisa em Hospital de Uberlândia, demonstrando contaminação de $40 \%$ das superfícies de enfermarias por $S$. aureus.
Um estudo realizado no Hospital Público de Goiás demonstrou uma variação de contaminação em superfícies inanimadas por E. coli de $25,59 \%$ a $32,56 \%$, sendo mais frequente no período chuvoso que na estiagem (FEITOSA et al., 2008)

As amostras avaliadas pertencem à área não crítica, que não são ocupadas por pacientes, como bancadas e depósitos; e semicríticas, que entramem contato com a pele não íntegra ou com mucosas íntegras (BRASIL, 1992).

Ao avaliar a porcentagem de contaminação das sete Unidades pelos locais analisados, constatou-se que somente uma UBS não apresentou contaminação de bioindicadores na superfície (Tabela 2). 
Tabela 2 - Frequência de biomarcadores nas Unidades analisadas.

\begin{tabular}{ccccc}
\hline UNIDADE & MICRO-ORGANIS MO & LOCAL & $\begin{array}{c}\text { VALOR } \\
\text { ABSOLUTO }\end{array}$ & PORCENTAGEM \\
\hline UNIDADE 1 & Staphylococcus aureus & Guichê da Recepção & $1 / 15$ & $6,7 \%$ \\
UNIDADE 2 & Staphylococcus aureus & Torneira da Sala de Curativos & $1 / 15$ & $13,3 \%$ \\
& Escherichia coli & Bebedouro & $1 / 15$ & $13,3 \%$ \\
UNIDADE 3 & Staphylococcus aureus & Torneira da Sala de Medicações & $2 / 15$ & $6,7 \%$ \\
UNIDADE 4 & Staphylococcus aureus & Bebedouro & $1 / 15$ & $20 \%$ \\
UNIDADE 5 & Staphylococcus aureus & Torneira da Sala de Curativos & $1 / 15$ & \\
& Escherichia coli & Maçaneta da Sala de Coleta & $1 / 15$ & - \\
UNIDADE 6 & - & Guichê da Farmácia & $1 / 15$ & - \\
UNIDADE 7 & Staphylococcus aureus & Guichê da Farmácia & $1 / 15$ & $6,7 \%$ \\
\hline
\end{tabular}

Fonte: Dados de pesquisa.

Acredita-se que a ausência dos patógenos pesquisados nas superfícies da UBS de maior complexidade se deva aos maiores cuidados em biossegurança, uma vez que são executados procedimentos hospitalares mais complexos. Nas outras UBS's, mesmo havendo baixa frequência dos patógenos nas superfícies estudadas, constata-se que há risco de contaminar pacientes e visitantes.

O S. aureus é considerado o patógeno humano mais importante do seu gênero, o Staphylococcus $S P$. As manifestações clínicas das doenças causadas por esse patógeno variam: intoxicações alimentares e infecções hospitalares graves, principalmente da corrente sanguínea, por ele ser secretor de exotoxinas e enzimas que podem também causar variedades de infecções cutâneas e sistêmicas, que incluem furúnculos, abscessos do choque tóxico e até a síndrome do choque tóxico neonatal. As infecções causada por esse patógeno acomete pacientes de todas as faixas etárias, mas com uma frequência maior em indivíduos acima de 50 anos. Por isso é de grande importância sua identificação e que se reconheçam os prováveis reservatórios para a avaliação da distribuição dessa espécie bacteriana, envolvida em complicações infecciosas de um estabelecimento de saúde, e assim promover o monitoramento de sua incidência (BROOKS et al., 2000; IWATSUKI et al., 2006;
KEIM, 2005; MOREIRA et al., 1998).

Os dados corroboram com pesquisa desenvolvida por Moreira (2002), que comprovou a presença de cepas do gênero Staphylococcus em três diferentes tipos de bancadas - mármore, granito e fórmica - em hospitais, podendo-se afirmar que em qualquer lugar é possívelencontrar microrganismos; no entanto, o fator que tira isso da normalidade é sua colonização demasiada e sua ligação com as IRAS, o que leva em consideração o fator da veiculação do profissional de saúde.

Mesmos os locais aparentemente limpos a olho nu podem estar contaminados, desde que haja uma pequena quantidade de material orgânico, sendo as mãos o principal veículo de contaminação cruzada. Dessa maneira, a correta lavagem e desinfecção das mãos já resolveria boa parte das contaminações das superfícies inanimadas. Além disso, é necessário que se tenha conhecimento dos vários agentes químicos, seus mecanismos de ação e aplicação (MOREIRA, 2002; YASSAKA et al., 2005).

A Portaria n. 930 (D.O.U. de 27/08/92), substituída pela Portaria 2616, de 12/5/1999, atualiza conceitos e normas do controle de infecção hospitalar, no seu anexo $\mathrm{V}$, métodos e produtos químicos para limpeza, desinfecção e esterilização de artigos e áreas em estabelecimentos de saúde do país. Para que 
alcance a eficácia no processo, há a necessidade de se detalhar prioridades, opções e considerações quanto ao tipo de carga microbiana depositada na superfície a ser processada, concentração de produtos, tempo de exposição, validade em uso e outros fatores relacionados (BRASIL, 1992).

Em superfícies como balcões do posto de enfermagem, deve-se fazer a desinfecção diariamente comálcoole depois álcool iodado, através do método de fricção, que é feito por meio da fricção mecânica com esponja, escova, vassoura, etc. Já para a limpeza deve-se usar água e sabão diariamente através do método de fricção com o uso de luvas. Em superfícies como bebedouros de esguicho, deve-se realizar a desinfecção e descontaminação diariamente com álcool através do método de fricção utilizando luvas, para a limpeza utiliza-se diariamente água e sabão através do método de fricção utilizando luvas (BRASIL, 1994).

\section{Conclusão}

Conclui-se, com o presente estudo, que as superfícies inanimadas podem apresentar importantes patógenos implicados nas IRAS. Isso pode ser demonstrado por meio da presença de biomarcadores que demonstram a deficiência na qualidade de desinfecção dessas superfícies.

Dessa maneira, é necessária a capacitação contínua dos profissionais da saúde sobre procedimentos de biossegurança, contribuindo na qualidade da saúde coletiva e na prevenção de doenças.

\section{REFERÊNCIAS}

ANDRADE, D.; ANGERAMI, E.L.S. \& PADOVANI, C.R. Condições Microbiológicas dos leitos hospitalares antes e depois de sua limpeza.

Revista de Saúde Pública, v.34, n.2, p.163-9, abr. 2000.

BOYCE, J.M.; POTTER-BYNOE, G.; CHENEVERT, C.; KING, T. Environmental contamination due to methicillin-resistant Staphylococcus aureus: possible infection control implications. Infection Control and Hospital

Epidemiology, v.18, n.9, p.622-627, sep. 1997.

BRASIL, Agência Nacional De Vigilância Sanitária. Portaria n. ${ }^{\circ} 930$ - Normas para o controle de infecções hospitalares. 1992.

BRASIL, Ministério Da Saúde. Coordenação de Controle de infecção Hospitalar. Processamento de artigos e superfícies em estabelecimentos de saúde. $2^{\mathrm{a}}$ ed. Brasília., 1994. 50p.

BRASIL. Agência Nacional de Vigilância Sanitária. Portaria n ${ }^{\circ}$ 2.616/MS/GM, de 12 de maio de 1998.

Diário Oficial da União, Brasília, 13 de maio de 1998.

BRASIL, Agência Nacional De Vigilância Sanitária. Detecção e Identificação de Bactérias de Importância Médica. Modulo V., 2004.

BROOKS, G.F.; BUTEL, J.S.; MORSE, S.A. Microbiologia médica. 21. ed. Rio de Janeiro: Guanabara Koogan, 2000.

CARVALHO, P.R. Boas práticas químicas em biossegurança. Rio de Janeiro: Interciência, 1999.

CARVALHO, K.S. Contaminação de superfícies em enfermarias de pacientes com infecções por Staphylococcus aureus no hospital de clínicas da universidade federal de Uberlândia, Uberlândia-MG. Uberlândia, MG: UFU. 47f.

Dissertação (Mestrado em Imunologia e

Parasitologia Aplicadas) - Universidade Federal de Uberlândia, Uberlândia. 2005.

FEITOSA, S. B.; ARAÚJO, R. B. de; COSTA, P. G. M. da; VIEIRA, J.; OLIVEIRA, M. B. R de; CARNEIRO, L.C.. Estudo de enterobactérias no hospital público de morrinhos-GO. SEMINÁRIO DE INICIAÇÃO CIENTÍFICA, 6., Resumos. Universidade Estadual de Goiás. Anápolis, 2008. 
Avaliação da qualidade do processo de desinfecção...

IWATSUKI, K.; YAMASAKI, O.; MORIZANE, S.; OONO T. Staphylococcal cutaneous infections: Invasion, evasion and aggression. Journal of Dermatological, v.42, n.3, p.203-214, 2006.

KEIM, L.S. Mapeamento dos estafilococos coagulase negativo no hospital universitário Antônio Pedro da Universidade Federal Fluminense, no período de 1998 a 2002 . Niterói, RJ: UFF, 2005.132 f. Dissertação (Mestrado em Ciências Médicas) - Universidade Federal Fluminense, Niterói. 2005.

MELO, S.C.O.; OLIVEIRA, R de C.B.W.; ARAÚJO, M.R.B. de. Isolamento e identificação de fungos oportunistas emunidades hospitalares nas cidades de Patos de Minas e Paracatu/MG. Revista Eletrônica Perquirere, v. 1, n.1, 13p. 2004.

MOREIRA, M.; MEDEIROS, E.A.S.; PIGNATARI, A.C.C.; WEY, S.B.; CARDO, D.M. Efeito da infecção hospitalar da corrente sanguínea por Staphylococcus 33 aureus resistente à oxacilina sobre a letalidade e o tempo de hospitalização.

Revista da Associação Médica Brasileira, v. 44, n. 4, p. 263- 268, 1998.

MOREIRA, L.R.C. Bancadas hospitalares: superfícies e porosidades como fontes potenciais de infecção. 115p. Tese (Mestrado em Bioengenharia) - Programa de Pós-Graduação do Instituto de Pesquisa e Desenvolvimento do Vale do Paraíba, Univap, São José dos Campos/SP, 2002.

MUNDIM, G.J.; DEZENA, R.A.; OLIVEIRA, A.C. S.; SILVA, P. R.; CARDOSO, M.;PEREIRA, G.A.; MORAIS, C.A.; TERRA, A.P.S. Avaliação da presença de Staphylococcus aureus nos leitos do Centro de Terapia Intensiva do Hospital Escola da Faculdade de Medicina do Triângulo Mineiro, em relação à posição no colchão antes e após a limpeza. Revista da Sociedade Brasileira de Medicina Tropical, v. 36, n. 6, p. 685-688, 2003.
OIE, S.; HOSOKAWA, I.; KAMIYA, A.

Contamination of room door handles bymethicillinsensitive/methicillin-resistant Staphylococcus aureus. Journal of Hospital Infection, v.51, n.2, p.140143, jun. 2002.

PRÈRE, M.F.; FAYET, O. A new genetic test for the rapid identification of shigatoxines producing (STEC), enteropathogenic (EPEC) E. coli isolates from children. Pathologie Biologie, Paris, v.53, p.466-469, 2005.

RABHAE, G.N; RIBEIRO-FILHO, N; FERNANDES, A.T. Infecção do Sítio Cirúrgico. In: Fernandes, A.T, Fernandes, M.O.V; RibeiroFilho, N. Infecção hospitalar e suas interfaces na área da saúde. São Paulo: Atheneu; p. 479505, 2000.

SCHEIDT, K.L.S.; ROSA, L.R.S.; LIMA, E.F.A. As Ações De Biossegurança Implementadas Pelas Comissões De Controle De Infecções Hospitalares. Rev Enferm. UERJ, Rio de Janeiro; v.14, n.3, p. 372-77, set. 2006.

\section{SEQUEIRA, E.J.D. Saúde ocupacional e} medidas de biossegurança. In: MARTINS, M.A. Manual de infecções hospitalares. 2. ed. Rio de Janeiro: Atheneu, 2001. p 643-73.

SHIOMORI, T.; MIYAMOTO, H.; MAKISHIMA, K.; YOSHIDA, M.; FUJIYOSHI, T.;UDAKA, T.; INABA, T.; HIRAKI, N.

Evaluation of bedmaking-related airbone and surface methicilin-resistant Staphilococcus aureus contamination. Journal of Hospital Infection, v.50, n.1, p.30-35, jan. 2002.

TEIXEIRA, P.; VALLE, S. Biossegurança: uma abordagem multidisciplinar. Rio de Janeiro: Fio Cruz, 1996. 362p.

YASSAKA, C.; OSPEDALL, K.Z.; MELLO R.A.; 
FUCCI et al.

PERISSUTTI, G.E. Análise da contaminação presente em ambientes de laboratório. 11.p, Iniciação científica. Universidade Tuiuti do Paraná. 2005.
WENZEL, R.P. Preoperative antibiotic prophylaxis. N Engl J Méd; v.5, p. 337-9, 1992.

ReCEBIDO EM 30/1/2013

ACEITO EM 22/5/2013 